\title{
The second-order contingent derivative of generalized perturbation maps
}

- Nguyen Le Hoang Anh

University of Science, VNU- HCMC

(Received on $5^{\text {th }}$ December 2016, accepted on $28^{\text {th }}$ November 2017)

\section{ABSTRACT}

In the paper, we give some remarks on [1]. Then, we modify main results concerning the sum rule of second-order contingent derivatives for set-valued maps and its application to the sensitivity analysis of

Key words: second-order proto-differentiability, second-order semi-differentiability, metric subregularity, generalized perturbation map, set-valued map

\section{INTRODUCTION}

In [1], the second-order proto-differentiability and second-order semi-differentiability for set-valued maps were firsthy discussed and applied to sum rules of two set-valued maps. Then, the authors established secondorder sensitivity analysis of generalized perturbation maps as an application of sum rules. The semidifferentiability plays an essential role in all main results in [1]. generalized perturbation maps. The obtained results are new and better than those in [1]. Some examples are proposed to illustrate our results.
In the paper, we give some remarks on the Proposition 2 and Theorem 1 in [1]. On the other hand, a new result is proposed to avoid the semidifferentiability by using a weaker hypothesis of the proto-differentiability.

The layout of this paper is as follows. Section 2 is devoted to several concepts needed in the sequel. Our main remarks and modified results are given in Section

\section{PRELIMINARIES} 3.

Throughout the paper, let $X$ and $Y$ be normed spaces. For a set-valued map $F: X \rightarrow 2^{Y}$, the domain, image, and graph of $F$ are defined, respectively (resp for short), by

$$
\begin{aligned}
& \operatorname{dom}(F):=\{x \in X \mid F(x) \neq \varnothing\}, \operatorname{im}(F):=\{y \in Y \mid y \in F(X)\}, \\
& \operatorname{gr}(F):=\{(x, y) \in X \times Y \mid y \in F(x)\} .
\end{aligned}
$$

Definition $2.1([2,3])$. Let $S \subseteq X, x \in \operatorname{cl}(S)$ and $w \in X$, where $\operatorname{cl}(S)$ denotes the closure of $S$.

(i) The contingent cone and the adjacent cone of $S$ at $x$ are defined by, resp,

$$
\begin{aligned}
T(S, x) & :=\left\{u \in X \mid \exists t_{n} \rightarrow 0^{+}, \exists u_{n} \rightarrow u, x+t_{n} u_{n} \in S\right\}, \\
T^{b}(S, x) & :=\left\{u \in X \mid \forall t_{n} \rightarrow 0^{+}, \exists u_{n} \rightarrow u, x+t_{n} u_{n} \in S\right\} .
\end{aligned}
$$


(ii) The second-order contingent cone and the second-order adjacent cone of $S$ at $x$ in the direction $w$ are defined by, resp,

$$
\begin{array}{r}
T^{2}(S, x, w):=\left\{u \in X \mid \exists t_{n} \rightarrow 0^{+}, \exists u_{n} \rightarrow u, x+t_{n} w+\frac{t_{n}^{2}}{2} u_{n} \in S\right\}, \\
T^{b(2)}(S, x, w):=\left\{u \in X \mid \forall t_{n} \rightarrow 0^{+}, \exists u_{n} \rightarrow u, x+t_{n} w+\frac{t_{n}^{2}}{2} u_{n} \in S\right\} .
\end{array}
$$

Remark 2.1. From the Observation 1 in [4], we obtain the equivalent formulae of Definition 2.1(ii) as follows

$$
\begin{gathered}
T^{2}(S, x, w):=\left\{u \in X \mid \begin{array}{l}
\exists \alpha_{n}, \beta_{n}>0: \alpha_{n} \rightarrow+\infty, \beta_{n} \rightarrow+\infty, \frac{\beta_{n}}{\alpha_{n}} \rightarrow 2, \\
\exists x_{n} \in S: \alpha_{n}\left(x_{n}-x\right) \rightarrow w, \beta_{n}\left(\alpha_{n}\left(x_{n}-x\right)-w\right) \rightarrow u
\end{array}\right\}, \\
T^{2(b)}(S, x, w):=\left\{u \in X \mid \begin{array}{l}
\forall \alpha_{n}, \beta_{n}>0: \alpha_{n} \rightarrow+\infty, \beta_{n} \rightarrow+\infty, \frac{\beta_{n}}{\alpha_{n}} \rightarrow 2, \\
\exists x_{n} \in S: \alpha_{n}\left(x_{n}-x\right) \rightarrow w, \beta_{n}\left(\alpha_{n}\left(x_{n}-x\right)-w\right) \rightarrow u
\end{array}\right\} .
\end{gathered}
$$

Definition 2.2 ([2,3]). Let $F: X \rightarrow 2^{Y},(x, y) \in \operatorname{gr}(F)$ and $(w, r) \in X \times Y$.

(i) The contingent derivative (the adjacent derivative) of $F$ at $(x, y)$ is a set-valued map $D F(x, y): X \rightarrow 2^{Y}$ ( $D^{b} F(x, y): X \rightarrow 2^{Y}$, resp) such that

$$
\begin{gathered}
\operatorname{gr}(D F(x, y)):=T(\operatorname{gr}(F),(x, y)) \\
\left(\operatorname{gr}\left(D^{b} F(x, y)\right):=T^{b}(\operatorname{gr}(F),(x, y)), \text { resp }\right) .
\end{gathered}
$$

(ii) The second-order contingent derivative (the second-order adjacent derivative) of $F$ at $(x, y)$ in the direction $(w, r)$ is a set-valued map $D^{2} F(x, y, w, r): X \rightarrow 2^{Y}\left(D^{2(b)} F(x, y, w, r): X \rightarrow 2^{Y}\right.$, resp) such that

$$
\begin{gathered}
\operatorname{gr}\left(D^{2} F(x, y, w, r)\right):=T^{2}(\operatorname{gr}(F),(x, y),(w, r)) \\
\left(\operatorname{gr}\left(D^{2(b)} F(x, y, w, r)\right):=T^{2(b)}(\operatorname{gr}(F),(x, y),(w, r)), \text { resp }\right) .
\end{gathered}
$$

Remark 2.2. From Definition 2.1 and Remark 2.1 is follous

$$
\begin{aligned}
& D^{2} F(x, y, w, r)(u)=\left\{v \in Y \mid \exists t_{n} \rightarrow 0^{+}, \exists u_{n} \rightarrow u, \exists v_{n} \rightarrow v, y+t_{n} r+\frac{t_{n}^{2}}{2} v_{n} \in F\left(x+t_{n} w+\frac{t_{n}^{2}}{2} u_{n}\right)\right\} \\
& =\left\{\begin{array}{l|l}
v \in Y & \begin{array}{l}
\exists \alpha_{n}, \beta_{n}>0: \alpha_{n} \rightarrow+\infty, \beta_{n} \rightarrow+\infty, \frac{\beta_{n}}{\alpha_{n}} \rightarrow 2, \\
\exists\left(x_{n}, y_{n}\right) \in \operatorname{gr}(F): \alpha_{n}\left(x_{n}-x\right) \rightarrow w, \beta_{n}\left(\alpha_{n}\left(x_{n}-x\right)-w\right) \rightarrow u, \\
\alpha_{n}\left(y_{n}-y\right) \rightarrow r, \beta_{n}\left(\alpha_{n}\left(y_{n}-y\right)-r\right) \rightarrow v
\end{array}
\end{array}\right\}, \\
& D^{b(2)} F(x, y, w, r)(u)=\left\{v \in Y \mid \forall t_{n} \rightarrow 0^{+}, \exists u_{n} \rightarrow u, \exists v_{n} \rightarrow v, y+t_{n} r+\frac{t_{n}^{2}}{2} v_{n} \in F\left(x+t_{n} w+\frac{t_{n}^{2}}{2} u_{n}\right)\right\}
\end{aligned}
$$




$$
=\left\{\begin{array}{l|l}
v \in Y & \begin{array}{l}
\forall \alpha_{n}, \beta_{n}>0: \alpha_{n} \rightarrow+\infty, \beta_{n} \rightarrow+\infty, \frac{\beta_{n}}{\alpha_{n}} \rightarrow 2, \\
\exists\left(x_{n}, y_{n}\right) \in g r(F): \alpha_{n}\left(x_{n}-x\right) \rightarrow w, \beta_{n}\left(\alpha_{n}\left(x_{n}-x\right)-w\right) \rightarrow u, \\
\alpha_{n}\left(y_{n}-y\right) \rightarrow r, \beta_{n}\left(\alpha_{n}\left(y_{n}-y\right)-r\right) \rightarrow v
\end{array}
\end{array}\right\} .
$$

Definition 2.3 ([5]). Let $F: X \rightarrow 2^{Y},(x, y) \in \operatorname{gr}(F)$ and $(w, r) \in X \times Y$. The second-order lower Dini derivative of $F$ at $(x, y)$ in direction $(w, r)$ is a set-valued map $D_{l}^{2} F(x, y, w, r): X \rightarrow 2^{Y}$ that is defined by

$$
D_{l}^{2} F(x, y, w, r)(u)=\left\{v \in Y \mid \forall t_{n} \rightarrow 0^{+}, \forall u_{n} \rightarrow u, \exists v_{n} \rightarrow v, y+t_{n} r+\frac{t_{n}^{2}}{2} v_{n} \in F\left(x+t_{n} w+\frac{t_{n}^{2}}{2} u_{n}\right)\right\} .
$$

Remark 2.3. (i) By the proof similar to that of Observation 1 in [4], we get

$$
D_{l}^{2} F(x, y, w, r)(u)=\left\{\begin{array}{l}
v \in Y \\
\forall x_{n} \in \operatorname{dom}(F): \alpha_{n}\left(x_{n}-x\right) \rightarrow w, \beta_{n}\left(\alpha_{n}\left(x_{n}-x\right)-w\right) \rightarrow u, \\
\exists y_{n} \in F\left(x_{n}\right): \alpha_{n}\left(y_{n}-y\right) \rightarrow r, \beta_{n}\left(\alpha_{n}\left(y_{n}-y\right)-r\right) \rightarrow v
\end{array}\right\} .
$$

(ii) It is obvious to see that

$D_{l}^{2} F(x, y, w, r)(u) \subseteq D^{b(2)} F(x, y, w, r)(u) \subseteq D^{2} F(x, y, w, r)(u)$.

Definition 2.4 ([6, 7]). Let $F: X \rightarrow 2^{Y}$, $(x, y) \in \operatorname{gr}(F)$ and $(w, r) \in X \times Y$.

(i) The map $F$ is said to be second-order protodifferentiable at $x$ relative to $y$ in the direction $(w, r)$ if $D^{2} F(x, y, w, r) \equiv D^{b(2)} F(x, y, w, r)$.

(ii) The map $F$ is said to be second-order semidifferentiable at $x$ relative to $y$ in the direction $(w, r)$ if $D^{2} F(x, y, w, r) \equiv D_{l}^{2} F(x, y, w, r)$.

It is easy to see that if $F$ is second-order semidifferentiable then $F$ is second-order protodifferentiable.

Definition 2.5 ([1]). Let $F: X \rightarrow 2^{Y},(x, y) \in \operatorname{gr}(F)$ and $(w, r) \in X \times Y$. The map $F$ is said to be second- order lower semi-differentiable at $(x, y)$ in the direction $(w, r)$ if for any $\alpha_{n}, \beta_{n}>0, x_{n} \in \operatorname{dom}(F)$ with $\quad \alpha_{n} \rightarrow+\infty, \beta_{n} \rightarrow+\infty, \frac{\beta_{n}}{\alpha_{n}} \rightarrow 2, \quad \alpha_{n}\left(x_{n}-x\right) \rightarrow w$ and $\beta_{n}\left(\alpha_{n}\left(x_{n}-x\right)-w\right) \rightarrow u$ for some $u \in X$, there exists a subsequence $y_{n_{i}} \in F\left(x_{n_{i}}\right)$ such that $\alpha_{n_{i}}\left(y_{n_{i}}-y\right) \rightarrow r \quad$ and $\quad \beta_{n_{i}}\left(\alpha_{n_{i}}\left(y_{n_{i}}-y\right)-r\right) \quad$ is convergent.

Remark 2.4. By Remark 2.3(i), if $F$ is second-order semi-differentiable then $F$ is second-order lower semidifferentiable. This assertion can be also implied immediately by Proposition 1 in [1].

Recall that a set-valued map $F: X \rightarrow 2^{Y}$ is called to be metric regular at $(x, y) \in \operatorname{gr}(F)$ if there are $\mu, r>0$ such that for all $u \in B_{X}(x, r), v \in B_{Y}(y, r)$, 


$$
d\left(u, F^{-1}(v)\right) \leq \mu d(v, F(u)),
$$

where $B_{X}(x, r)$ denotes the open ball in $X$ centered at $x$ with radius $r$.

By fixing $v=y$ in (1), we get a weaker definition named by metric subregularity. The metric (sub)regularity plays an important role in variational analysis and has been applied to many topics of optimization, see [2, 8-10] and the references therein.

Inspired of the above definition, we propose the following concept.

Definition 2.6. Let $F: X \rightarrow 2^{Y}, \quad(x, y) \in \operatorname{gr}(F)$ and $S \subseteq X$. The map $F$ is said to be metric subregularity at $(x, y) \in \operatorname{gr}(F)$ with respect to $S$ if there are $\mu, r>0$ such that for all $u \in B_{X}(x, r) \cap S, v \in B_{Y}(y, r)$,

$$
d\left(u, F^{-1}(y) \bigcap S\right) \leq \mu d(y, F(u)) .
$$

\section{MAIN RESULTS} follows.

Firstly, we recall a main proposition in [1] as

Proposition 3.1 (Proposition 2 in [1]). Suppose that $\bar{G}: X \rightarrow 2^{Y}$ is second-order proto-differentiable at $x$ relative to $\bar{y} \in \bar{G}(x)$ in the direction $(w, \bar{r}) \in X \times Y$, $G: X \rightarrow 2^{Y}$ is second-order semi-differentiable at $x$ relative to $y \in G(x)$ in the direction $(w, \hat{r}) \in X \times Y$ and $G$ is second-order lower semi-differentiable at $(x, y)$ in the direction $(w, \hat{r})$. Then, the set-valued map $G: X \rightarrow 2^{Y}$ defined by $G:=\bar{G}+G$ is secondorder proto-differentiable at $x$ relative to $y:=\bar{y}+y$ in the direction $(w, r)$, where $r:=\bar{r}+\hat{r}$, and for all $u \in X$,

$D^{2} G(x, y, w, r)(u)=D^{2} \bar{G}(x, \bar{y}, w, \bar{r})(u)+D^{2} G(x, y, w, \hat{r})(u)$

Remark 3.1. (i) From Remark 2.4, the assumption on the second-order lower semi-differentiability of $G$ at $(x, y)$ in the direction $(w, \hat{r})$ is superfluous. (ii) In the proof of the Proposition 2 (see page 248 of [1]), the authors implied that $v-\hat{v} \in D^{2} \bar{G}(x, \bar{y}, w, \bar{r})(u)$ from the second-order lower semi-differentiability of $G$. This assertion is right if $\overline{y_{n}} \in \bar{G}\left(x_{n}\right)$. Since

$$
\overline{y_{n}}=y_{n}-y_{n} \in G\left(x_{n}\right)-G\left(x_{n}\right)=\bar{G}\left(x_{n}\right)+G\left(x_{n}\right)-G\left(x_{n}\right)
$$

and $G$ is a set-valued map, it is not sure that $\overline{y_{n}}$ belongs to $\bar{G}\left(x_{n}\right)$. Thus, the conclusion of the Proposition 3.1 may be not true, see Example 2.1 in [11]. follows.

Hence, the Proposition 3.1 should be presented as

Proposition 3.2. Let $\bar{G}, G: X \rightarrow 2^{Y}$, $(x, \bar{y}) \in \operatorname{gr}(\bar{G}),(x, y) \in \operatorname{gr}(G)$ and $(w, \bar{r}, \hat{r}) \in X \times Y \times Y$

. Suppose that $G$ is second-order semi-differentiable at $x$ relative to $y$ in the direction $(w, \hat{r})$. Then, for all $u \in X$,

$D^{2} \bar{G}(x, \bar{y}, w, \bar{r})(u)+D^{2} G(x, y, w, \hat{r})(u) \subseteq D^{2} G(x, y, w, r)(u)$,

where $G:=\bar{G}+G, y:=\bar{y}+y$ and $r:=\bar{r}+\hat{r}$.

When $G$ is a single-valued map, (2) become an equality. If, additionally, $\bar{G}$ is second-order protodifferentiable at $x$ relative to $\bar{y}$ in the direction $(w, \bar{r})$ , then $G$ is second-order proto-differentiable at $x$ relative to $y$ in the direction $(w, r)$.

Proof It is similar to the proof of Proposition 2 in [1].

As an application of Proposition 3.1 to sensitivity analysis of generalized perturbation maps, Theorem 1 in [1] should be stated by

Theorem 3.1. Let $X, Y, Z$ be normed spaces, $G: X \times Z \rightarrow 2^{Y}$ be defined by $G(x, z):=\{y \in D \mid z \in F(x, y)+K(y)\}$, where $D$ is a closed convex subset in $Y, \quad F: X \times Y \rightarrow 2^{Z}$,

\section{Trang 206}


$K: D \rightarrow 2^{Z},(y, \bar{z}) \in \operatorname{gr}(K),((x, y), \bar{z}-z) \in \operatorname{gr}(F)$ and $\quad z-\bar{z}$ in the direcction $(w, r, q-\bar{q})$. Then, for all $u \in X$ $(w, r, q-\bar{q}) \in X \times(D-y) \times Z$. Suppose that $F$ is ,

second-order semi-differentiable at $(x, y)$ relative to

$$
\begin{aligned}
&\left\{v \in T^{2}(D, y, r) \mid\right.\left.p \in D^{2} F(x, y, z-\bar{z}, w, r, q-\bar{q})(u, v)+D^{2} K(y, \bar{z}, r, \bar{q})(v)\right\} \\
& \subseteq D^{2} G(x, z, y, w, q, r)(u, p) .
\end{aligned}
$$

When $F$ is a single-valued map, (3) is an equality. If, additionally, $K$ is second-order proto-differentiable at $y$ relative to $\bar{z}$ in the direction $(r, \bar{q})$, then $G$ is second-order proto-differentiable at $(x, z)$ relative to $y$ in the direction $(w, q, r)$.

Proof. The reader is referred to Theorem 1 in [1].

A natural question arises: which conditions ensure that (3.1) becomes an equality when all maps are setvalued? To get the answer, we recall a concept of the TP-derivative (see [12]) of a set-valued map $F: X \rightarrow 2^{Y}$ at $(x, y) \in \operatorname{gr}(F)$ as follows

$$
D_{T P} F(x, y)(u):=\left\{v \in Y \mid \exists t_{n}>0, \exists\left(u_{n}, v_{n}\right) \rightarrow(u, v): x+t_{n} u_{n} \rightarrow x, y+t_{n} v_{n} \in F\left(x+t_{n} u_{n}\right)\right\} .
$$

The following concept is necessary for our next result.

Definition 3.1. Let $F: X \rightarrow 2^{Y},(x, y) \in g r(F)$ and $(w, r) \in X \times Y$. The asymptotic second-order TP-derivative of $F$ at $(x, y)$ in the direction $(w, r)$ is a set-valued map $D_{T P}^{2} F(x, y, w, r): X \rightarrow 2^{Y}$ defined by

$$
D_{T P}^{2} F(x, y, w, r)(u):=\left\{v \in Y \mid \begin{array}{l}
\exists t_{n}, h_{n}>0, \exists\left(u_{n}, v_{n}\right) \rightarrow(u, v): x+t_{n} w+\frac{t_{n} h_{n}}{2} u_{n} \rightarrow x, \\
y+t_{n} r+\frac{t_{n} h_{n}}{2} v_{n} \in F\left(x+t_{n} w+\frac{t_{n} h_{n}}{2} u_{n}\right)
\end{array}\right\} .
$$

It is obvious to see that $D_{T P}^{2} F(x, y, 0,0)(u)=D_{T P} F(x, y)(u)$ for all $u \in X$. By virtue of the asymptotic secondorder TP-derivative, we obtain the converse inclusion of (3.1) for set-valued maps as follows.

Proposition 3.3. Let $\bar{G}, G: X \rightarrow 2^{Y},(x, \bar{y}) \in \operatorname{gr}(\bar{G}),(x, y) \in \operatorname{gr}(G)$ and $(w, \bar{r}, \hat{r}) \in X \times Y \times Y$. Suppose that $Y$ is finite dimensional and

$$
D_{T P}^{2} \bar{G}(x, \bar{y}, w, \bar{r})(0) \bigcap\left(-D_{T P}^{2} G(x, y, w, \hat{r})(0)\right)=\{0\} .
$$

Then, for all $u \in X$,

$$
D^{2} G(x, y, w, r)(u) \subseteq D^{2} \bar{G}(x, \bar{y}, w, \bar{r})(u)+D^{2} G(x, y, w, \hat{r})(u),
$$

where $G:=\bar{G}+G, y:=\bar{y}+y$ and $r:=\bar{r}+\hat{r}$.

Proof. Let $v \in D^{2} G(x, y, w, r)(u)$, then there exist $t_{n} \rightarrow 0^{+},\left(u_{n}, v_{n}\right) \rightarrow(u, v)$ such that

$$
y+t_{n} r+\frac{t_{n}^{2}}{2} v_{n} \in G\left(x+t_{n} w+\frac{t_{n}^{2}}{2} u_{n}\right)=\bar{G}\left(x+t_{n} w+\frac{t_{n}^{2}}{2} u_{n}\right)+G\left(x+t_{n} w+\frac{t_{n}^{2}}{2} u_{n}\right) .
$$


Thus, there are $\overline{y_{n}} \in \bar{G}\left(x+t_{n} w+\frac{t_{n}^{2}}{2} u_{n}\right)$ and $y_{n} \in G\left(x+t_{n} w+\frac{t_{n}^{2}}{2} u_{n}\right)$ such that

$$
v_{n}=\overline{v_{n}}+v_{n},
$$

where $\overline{v_{n}}:=\frac{\overline{y_{n}}-\bar{y}-t_{n} \bar{r}}{(1 / 2) t_{n}^{2}}, v_{n}:=\frac{y_{n}-y-t_{n} \hat{r}}{(1 / 2) t_{n}^{2}}$. We now prove that the sequence $v_{n}$ has a convergent subsequence. Suppose to the contrary, i.e., $\left\|v_{n}\right\| \rightarrow+\infty$. By setting $z_{n}:=v_{n} /\left\|v_{n}\right\|$, then $z_{n}$ (taking a subsequence if necessary) has a limit point $\hat{z}$ with $\|\hat{z}\|=1$. Moreover,

$$
y+t_{n} \hat{r}+\frac{t_{n}\left(t_{n}\left\|v_{n}\right\|\right)}{2} z_{n}=y_{n} \in G\left(x+t_{n} w+\frac{t_{n}\left(t_{n}\left\|v_{n}\right\|\right)}{2}\left(\frac{u_{n}}{\left\|v_{n}\right\|}\right)\right) .
$$

Let $h_{n}:=t_{n}\left\|v_{n}\right\|$, we get $\hat{z} \in D_{T P}^{2} G(x, y, w, \hat{r})(0)$. It follows from (5) that

$$
\frac{v_{n}}{\left\|v_{n}\right\|}=\frac{\overline{v_{n}}}{\left\|v_{n}\right\|}+\frac{v_{n}}{\left\|v_{n}\right\|}
$$

which implies $\overline{v_{n}} /\left\|v_{n}\right\| \rightarrow-\hat{z}$. On the other hand,

$$
\bar{y}+t_{n} \bar{r}+\frac{t_{n}\left(t_{n}\left\|v_{n}\right\|\right)}{2}\left(\frac{\bar{v}_{n}}{\left\|v_{n}\right\|}\right)=\overline{y_{n}} \in \bar{G}\left(x+t_{n} w+\frac{t_{n}\left(t_{n}\left\|v_{n}\right\|\right)}{2}\left(\frac{u_{n}}{\left\|v_{n}\right\|}\right) .\right.
$$

Thus, $-\hat{z} \in D_{T P}^{2} \bar{G}(x, \bar{y}, w, \bar{r})(0)$, which contradicts (4). Without loss of generality, we assume that $v_{n}$ converges to $\hat{v}$, then $\hat{v} \in D^{2} G(x, y, w, \hat{r})(u)$. From (5), one gets $\quad \overline{v_{n}}=v_{n}-v_{n} \rightarrow v-\hat{v}$. Hence $v-\hat{v} \in D^{2} \bar{G}(x, \bar{y}, w, \bar{r})(u)$, i.e., $v \in D^{2} \bar{G}(x, \bar{y}, w, \bar{r})(u)+D^{2} G(x, y, w, \hat{r})(u)$.

Note that (3.3) is only a sufficient condition (not necessary condition) for the converse inclusion of (2), see Example 2 in [1].

It follows from the Proposition 3.3 that the Proposition 3.2 and the Theorem 3.1 can be modified for set-valued maps as follows.
Proposition 3.4. Let $Y$ be finite dimensional, $\bar{G}, G: X \rightarrow 2^{Y}, \quad(x, \bar{y}) \in \operatorname{gr}(\bar{G}),(x, y) \in \operatorname{gr}(G), \quad$ and $(w, \bar{r}, \hat{r}) \in X \times Y \times Y$. Suppose that $G$ is second-order semi-differentiable at $x$ relative to $y$ in the direction $(w, \hat{r})$ and (4) holds. Then, (2) becomes an equality. If, additionally, $\bar{G}$ is second-order proto-differentiable at $x$ relative to $\bar{y}$ in the direction $(w, \bar{r})$, then $G$ is second-order proto-differentiable at $x$ relative to $y$ in the direction $(w, r)$.

Theorem 3.2. Let $Y$ be finite dimensional, $G: X \times Z \rightarrow 2^{Y}$ be defined by $G(x, z):=\{y \in D \mid z \in F(x, y)+K(y)\}$, where $D$ is a closed convex subset in $Y, \quad F: X \times Y \rightarrow 2^{Z}$,

\section{Trang 208}


$K: D \rightarrow 2^{Z},(y, \bar{z}) \in \operatorname{gr}(K),((x, y), \bar{z}-z) \in \operatorname{gr}(F)$

and $(w, r, q-\bar{q}) \in X \times(D-y) \times Z$. Suppose that $F$ is second-order semi-differentiable at $(x, y)$ relative to $z-\bar{z}$ in the direcction $(w, r, q-\bar{q})$ and $D_{T P}^{2} F(x, y, z-\bar{z}, w, r, q-\bar{q})(0,0) \bigcap\left(-D_{T P}^{2} K(y, \bar{z}, r, \bar{q})(0)\right)=\{($

Then, (3) is an equality. If, additionally, $K$ is secondorder proto-differentiable at $y$ relative to $\bar{z}$ in the direction $(r, \bar{q})$, then $G$ is second-order protodifferentiable at $(x, z)$ relative to $y$ in the direction $(w, q, r)$.

By taking $(w, r)=(0,0)$, the Proposition 3.4 and the Theorem 3.2 were reduced to Proposition 2.1 and the Theorem 3.1 in [11], respectively.

In [1], the second semi-differentiability is employed to get (3.1). Although it is a quite strict condition, this concept (or relative versions) is used in

$$
\bar{y}+t_{n} \bar{r}+\frac{t_{n}^{2}}{2} \overline{v_{n}} \in \bar{G}\left(x+t_{n} w+\frac{t_{n}^{2}}{2} \overline{u_{n}}\right)
$$

Since $G$ is second-order proto-differentiable at $x$ relative to $y$ in the direction $(w, \hat{r})$, with $t_{n}$ above, there are $\left(u_{n}, v_{n}\right) \rightarrow(u, \hat{v})$ such that

$$
y+t_{n} \hat{r}+\frac{t_{n}^{2}}{2} v_{n} \in G\left(x+t_{n} w+\frac{t_{n}^{2}}{2} u_{n}\right)
$$

According to the metric subregularity of $g$, there exist $\mu, \lambda>0$ such that for every $\left(u_{1}, v_{1}, u_{2}, v_{2}\right) \in$

$$
\begin{aligned}
\left(B_{X \times Y}((x, \bar{y}), \lambda) \times B_{X \times Y}((x, y), \lambda)\right) \cap(\operatorname{gr}(\bar{G}) \times g r(G)) & \\
& d\left(\left(u_{1}, v_{1}, u_{2}, v_{2}\right), g^{-1}\left(0_{X}\right) \cap(\operatorname{gr}(\bar{G}) \times g r(G))\right) \leq \mu d\left(0_{X}, g\left(u_{1}, v_{1}, u_{2}, v_{2}\right)\right) .
\end{aligned}
$$

For $n$ large enough, we have

$$
\left(x+t_{n} w+\frac{t_{n}^{2}}{2} \overline{u_{n}}, \bar{y}+t_{n} \bar{r}+\frac{t_{n}^{2}}{2} \overline{v_{n}}, x+t_{n} w+\frac{t_{n}^{2}}{2} u_{n}, y+t_{n} \hat{r}+\frac{t_{n}^{2}}{2} v_{n}\right) \in B_{X \times Y}((x, \bar{y}), \lambda) \times B_{X \times Y}((x, y), \lambda) .
$$

Thus, it follows from (3.5) that there exists $\left(\bar{x}_{n}, \bar{y}_{n}, x_{n}, y_{n}\right) \in \operatorname{gr}(\bar{G}) \times \operatorname{gr}(G)$ with $\overline{x_{n}}=x_{n}$ for all $n$ such that 


$$
\left\|(x, \bar{y}, x, y)+t_{n}(w, \bar{r}, w, \hat{r})+\frac{t_{n}^{2}}{2}\left(\overline{u_{n}}, \overline{v_{n}}, u_{n}, v_{n}\right)-\left(\overline{x_{n}}, \overline{y_{n}}, x_{n}, y_{n}\right)\right\| \leq \mu \frac{t_{n}^{2}}{2}\left\|\overline{u_{n}}-u_{n}\right\|,
$$

which implies

$$
\begin{aligned}
& \left\|\left(\bar{y}+t_{n} \bar{r}+\frac{t_{n}^{2}}{2} \overline{v_{n}}-\overline{y_{n}}\right)+\left(y+t_{n} \hat{r}+\frac{t_{n}^{2}}{2} v_{n}-y_{n}\right)\right\| \\
\leq & \left\|\bar{y}+t_{n} \bar{r}+\frac{t_{n}^{2}}{2} \overline{v_{n}}-\overline{y_{n}}\right\|+\left\|y+t_{n} \hat{r}+\frac{t_{n}^{2}}{2} v_{n}-y_{n}\right\| \\
\leq & 2\left\|(x, \bar{y}, x, y)+t_{n}(w, \bar{r}, w, \hat{r})+\frac{t_{n}^{2}}{2}\left(\overline{u_{n}}, \overline{v_{n}}, u_{n}, v_{n}\right)-\left(\overline{x_{n}}, \overline{y_{n}}, x_{n}, y_{n}\right)\right\| \\
\leq & \mu t_{n}^{2}\left\|\bar{u}_{n}-u_{n}\right\|
\end{aligned}
$$

and

$$
\left\|x+t_{n} w+\frac{t_{n}^{2}}{2} \overline{u_{n}}-\overline{x_{n}}\right\| \leq\left\|(x, \bar{y}, x, y)+t_{n}(w, \bar{r}, w, \hat{r})+\frac{t_{n}^{2}}{2}\left(\overline{u_{n}}, \overline{v_{n}}, u_{n}, v_{n}\right)-\left(\overline{x_{n}}, \overline{y_{n}}, x_{n}, y_{n}\right)\right\| \leq \mu \frac{t_{n}^{2}}{2}\left\|\overline{u_{n}}-u_{n}\right\| .
$$

Thus,

$$
\left\|\frac{\left(\overline{y_{n}}+y_{n}\right)-(\bar{y}+y)-t_{n}(\bar{r}+\hat{r})}{(1 / 2) t_{n}^{2}}-\left(\overline{v_{n}}+v_{n}\right)\right\| \leq 2 \mu\left\|\overline{u_{n}}-u_{n}\right\|
$$

and

$$
\left\|\frac{\overline{x_{n}}-x-t_{n} w}{(1 / 2) t_{n}^{2}}-\overline{u_{n}}\right\| \leq \mu\left\|\overline{u_{n}}-u_{n}\right\|
$$

Setting

$$
v_{n}:=\frac{\left(\overline{y_{n}}+y_{n}\right)-(\bar{y}+y)-t_{n}(\bar{r}+\hat{r})}{(1 / 2) t_{n}^{2}}, u_{n}:=\frac{\overline{x_{n}}-x-t_{n} w}{(1 / 2) t_{n}^{2}},
$$

then $v_{n} \rightarrow \bar{v}+\hat{v}, u_{n} \rightarrow u$ (let $n \rightarrow+\infty$ in (7) and (8) and

$$
y+t_{n} r+\frac{t_{n}^{2}}{2} v_{n}=\overline{y_{n}}+y_{n} \in \bar{G}\left(\overline{x_{n}}\right)+G\left(x_{n}\right)=G\left(x+t_{n} w+\frac{t_{n}^{2}}{2} \overline{u_{n}}\right),
$$

where $y:=\bar{y}+y$ and $r:=\bar{r}+\hat{r}$. Hence, $\bar{v}+\hat{v} \in D^{2} G(x, y, w, r)(u)$.

Taking $(w, \bar{r}, \hat{r})=(0,0,0)$, the Proposition 3.5 reduces to the following result. 
Corollary 3.1. Let $\bar{G}, G: X \rightarrow 2^{Y},(x, \bar{y}) \in \operatorname{gr}(\bar{G}),(x, y) \in \operatorname{gr}(G)$. Suppose that $G$ is proto-differentiable at $x$ relative to $y$ and the map $g:(X \times Y)^{2} \rightarrow X$ defined as in Proposition 3.5 is metric subregular at $\left(x, \bar{y}, x, y, 0_{X}\right)$ with respect to $\operatorname{gr}(\bar{G}) \times \operatorname{gr}(G)$. Then,

$$
D \bar{G}(x, \bar{y})(u)+D G(x, y)(u) \subseteq D(\bar{G}+G)(x, \bar{y}+y)(u) .
$$

Corollary 3.1 can be used to replace the semi-differentiability of $G$ in Proposition 2.1 in [11].

Our condition on the metric subregularity in the Proposition 3.5 is very different from the semi-differentiability condition. However, the following example shows a case where the Corollary 3.1 works, while the Proposition 3.2 does not.

Example 3.1. Let $\bar{G}, G: \mathbb{R} \rightarrow 2^{\mathbb{R}}$ be defined by

$$
\bar{G}(x)=G(x):=\left\{\begin{array}{l}
\varnothing, x \in\left\{\frac{1}{n^{2}} \mid n \in \mathbb{N}\right\}, \\
\{x\}, \text { otherwise. }
\end{array}\right.
$$

Then, we have

$$
(\bar{G}+G)(x):=\left\{\begin{array}{l}
\varnothing, x \in\left\{\frac{1}{n^{2}} \mid n \in \mathbb{N}\right\} \\
\{2 x\}, \text { otherwise. }
\end{array}\right.
$$

By calculating, one gets

$$
D G(0,0)(u)=D^{b} G(0,0)(u)=\{u\}, D_{l} G(0,0)(0)=\varnothing,
$$

which implies that $G$ is proto-differentiable at 0 relative to 0 , but it is not semi-differentiable at 0 relative to 0 . Thus, Proposition 3.2 cannot be employed in this example. However, the metric subregularity of Corollary 3.1 is fulfilled. Indeed, let $\lambda>0$, we need to show that there exists $\mu>0$ such that for all $\left(\bar{u}, \bar{v}^{\prime}, u, \hat{v}^{\prime}\right) \in\left(B_{\mathbb{R}^{2}}((0,0), \lambda) \times B_{\mathbb{R}^{2}}((0,0), \lambda)\right) \cap(\operatorname{gr}(\bar{G}) \times \operatorname{gr}(G))$,

$$
d\left(\left(\bar{u}, \bar{v}^{\prime}, u, \hat{v}^{\prime}\right), g^{-1}(0) \cap(\operatorname{gr}(\bar{G}) \times \operatorname{gr}(G))\right) \leq \mu d\left(0, g\left(\bar{u}, \bar{v}^{\prime}, u, \hat{v}^{\prime}\right)\right) .
$$

Since $\left(\bar{u}, \bar{v}^{\prime}, u, \hat{v}^{\prime}\right) \in \operatorname{gr}(\bar{G}) \times \operatorname{gr}(G)$, we get $\bar{u}=\bar{v}^{\prime}$ and $u=\hat{v}^{\prime}$. Thus, it is enough to find $\mu>0$ such that

$$
\inf _{x \in \mathbb{R},(\bar{y}, y) \in(\bar{G} \times G)(x)}\left\{|\bar{u}-x|+\left|\overline{v^{\prime}}-\bar{y}\right|+|u-x|+\left|\hat{v}^{\prime}-y\right|\right\} \leq \mu|\bar{u}-u| .
$$

On the other hand, we have

$$
\inf _{x \in \mathbb{R},(\bar{y}, y) \in(\bar{G} \times G)(x)}\left\{|\bar{u}-x|+\left|\overline{v^{\prime}}-\bar{y}\right|+|u-x|+\left|\hat{v}^{\prime}-y\right|\right\}=2 \inf _{x \in \mathbb{R}}\{|\bar{u}-x|+|u-x|\} .
$$

Taking $x=\frac{\bar{u}+u}{2}$, then we get 


$$
2 \inf _{x \in \mathbb{R}}\{|\bar{u}-x|+|u-x|\} \leq 2\left(\left|\bar{u}-\frac{\bar{u}+u}{2}\right|+\left|u-\frac{\bar{u}+u}{2}\right|\right)=2|\bar{u}-u| .
$$

Thus, (9) is true for every $\mu \geq 2$. Hence, by Corollary 3.1 , we get

$$
D \bar{G}(0,0)(u)+D G(0,0)(u) \subseteq D(\bar{G}+G)(0,0)(u) .
$$

\section{CONCLUSION}

In the paper, we propose remarks for some results in [1]. Then, by virtue of the proto-differentiability, a weaker hypothesis than the semi-differentiability introduced in [1], we obtain a new result on second- order sensitivity analysis of generalized perturbation maps.

Acknowledgment: The author acknowledges financial support from University of Science, VNU-HCM (under grant number T2016-01).

\section{Đạo hàm contingent cấp hai của ánh xạ nhiễu suy rộng}

- Nguyễn Lê Hoàng Anh

Trường Đại học Khoa học Tự nhiên, ĐHQG -HCM

\section{TÓM TẮT}

Trong bài báo này, chúng tôi đura ra một số nhận xét về các kết quả của [1]. Sau đó, chúng tôi phát triển các kết quả chính liên quan đến phép tổng của đạo hàm contingent cấp hai cho ánh xa đa trị và các áp dụng trong phân tích độ nhạy của ánh xạ nhiễu suy rộng. Kết quả đạt được là mói và cải thiện các kết quả của [1]. Một số ví du minh hoạ cũng được đưa ra.

Tù khoá: Tiền khả vi cấp hai, nưa khả vi cấp hai, chính quy duới metric, ánh xạ nhiễu suy rộng, ánh xạ đa trị

\section{REFERENCES}

[1]. S.J. Li, C.M. Liao, Second-order differentiability of generalized perturbation maps, Journal of Global Optimization, 52, 243-252 (2012).

[2]. J.P., Aubin, H. Frankowska, Set-Valued Analysis, Birkhauser, Boston (1990).

[3]. J. Jahn, A.A. Khan, P. Zeilinger, Second-order optimality conditions in set-optimization, Journal of Optimization Theory and Applications, 125, 331347 (2005).

[4]. A. Cambini A., L. Martein L., M. Vlah, Second order tangent sets and optimality conditions, Mathematica Japonica, 49, 451-461 (1999).
[5]. V. Kalashnikov, B. Jadamba B., A.A. Khan, First and second-order optimality conditions in set Optimization, in: S. Dempe, V. Kalashnikov (eds) Optimization with Multivalued Mappings, 265-276 (2006).

[6]. J.P. Penot, Differentiability of relations and differential stability of perturbed optimization problems, SIAM Journal of Control Optimization, 22, 529-551 (1984).

[7]. R.T. Rockafellar, Proto-differentiability of setvalued mappings and its applications in 
optimization, Annales De L'Institut Henri PoincaréAnalyse Non Linéaire, 6, 449-482 (1989).

[8]. B.S. Mordukhovich, Variational Analysis and Generalized Differentiation, Vol.I Basic Theory, Springer, Berlin (2006).

[9]. B.S. Mordukhovich, Variational Analysis and Generalized Differentiation, Vol.II Applications, Springer, Berlin (2006).

[10]. R.T. Rockafellar, R.J.B. Wets, Variational Analysis, 3rd edn, Springer, Berlin (2009).

[11]. G.M. Lee, N.Q. Huy, On proto differentiability of generalized perturbation maps, Journal of Mathematical Analysis and Applications, 324, 1297-1309 (2006).
[12]. D.S. Shi, Contingent derivative of the perturbation map in multiobjective optimization, Journal of Optimization Theory and Applications, 70, 385-396 (1991).

[13]. N.L.H. Anh, P.Q. Khanh, L.T. Tung, Variational sets : calculus and applications to nonsmooth vector Optimization, Nonlinear Analysis: Theory, Methods and Applications, 74, 2358-2379 (2011).

[14]. N.L.H. Anh, P.Q. Khanh, Higher-order optimality conditions in set-valued optimization using radial sets and radial derivatives, Journal of Global Optimization, 56, 519-536 (2013).

[15]. N.L.H. Anh, P.Q. Khanh, Calculus and applications of Studniarski's derivatives to sensitivity and implicit function theorems, Control and Cybernetics, 43, 34-57 (2014).

[16]. S.J. Li, K.W. Meng, J.P. Penot, Calculus rules for derivatives of multimaps, Set-Valued and Variational Analysis, 17, 21-39 (2009). 